\title{
Analyse des variations de pressions générées par la houle sur la digue Jarlan du port de Dieppe
}

\author{
Jean-Marc Rousset \\ Doctorant, Laboratoire de Mécanique, Université de Caen
}

\author{
Michel Bélorgey \\ Professeur, Laboratoire de Mécanique, Université de Caen
}

Résumé

La conception des digues verticales nécessite la détermination des forces exercées par la houle sur ces structures, actuellement calculées par les formules de Goda. Dans le but de mieux adapter ces lois aux caissons perforés, nous avons procédé à l'acquisition de pressions subies par un caisson Jarlan de la digue de Dieppe. Il apparaît que les sous-pressions du radier ne suivent pas les prévisions: distribution non-triangulaire et valeurs faibles. Un déphasage des pressions existe de part et d'autre de la paroi perforée. Celle-ci agit par ailleurs comme un filtre fréquentiel de l'onde incidente. Ces premiers résultats prouvent l'intérêt de confronter les modèles à la réalité du milieu naturel, malgré les difficultés de mise en œuvre de cette démarche.

\section{Introduction}

L'utilisation de jetées verticales formées de caissons parallélépipèdiques est une alternative avantageuse à la mise en place de digues enrochements dans les zones à marée. Il est par contre primordial pour la sûreté de tels ouvrages de déterminer les valeurs des forces exercées par la houle. La formule de Goda (1985) est recommandée pour les calcuis concernant les caissons pleins verticaux. Son adaptation aux caissons perforés est nécessaire afin de connaître les forces de basculement, de glissement et les contraintes appliquées à la paroi perforée indispensables à son bon dimensionnement.

Les modélisations physiques et numériques représentent une approche simplifiée des phénomènes mis en jeu. Toutefois il est indispensable de confronter ces résultats avec la réalité, c'est à dire avec des données provenant de digues perforées instrumentées.

Nous présentons ici les premières analyses des pressions enregistrées sur la digue Jarlan de Dieppe.

\section{Description de la digue et de l'instrumentation}

\subsection{Description de la digue}

La digue ouest de 1'avant-port de Dieppe est constituée de neuf double caissons Jarlan en béton armé (huit caissons rectangulaires et un musoir arrondi). Elle est orientée dans l'axe sud-nord et prolonge l'ancienne digue ouest. D'une longueur totale de $225 \mathrm{~m}$, la jetée repose sur des fondations comprenant un noyau en tout- 
venant $(80 \mathrm{~mm})$, une surface de friction en galets $(20$ à $63 \mathrm{~mm})$ et une carapace en rochers ( 1 à 3 tonnes). Il est à noter qu'un lit de fascines a été déposé sous les caissons afin d'amortir leur immersion et éviter la dispersion des galets. Un schéma du caisson est présenté sur la figure 1.

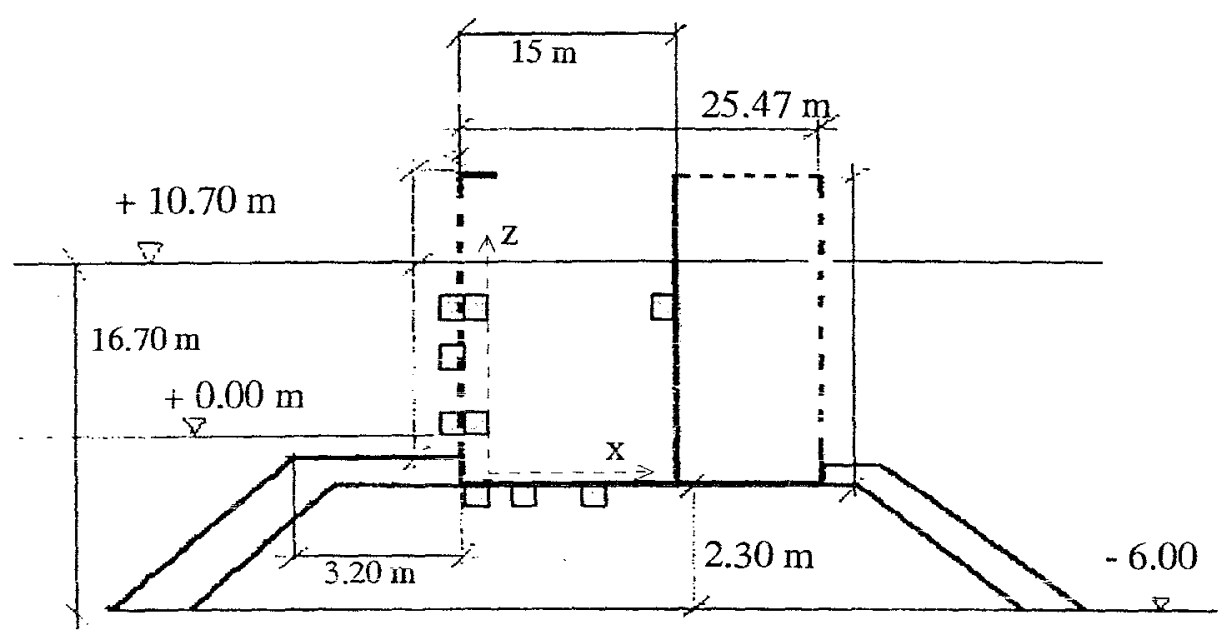

Figure 1: Schéma du caisson Jarlan et des capteurs.

\subsection{Instrumentation du caisson}

L'instrumentation du caisson $n^{\circ} 6$ a débuté lors de sa construction dans un des bassins du port du Havre en 1991. Elle a consisté en la pose de sept capteurs de pression TransAmerica XL1F sous le radier. Après la mise en place de la jetée à Dieppe, seules des acquisitions ponctuelles des pressions ont été réalisées.

Durant l'année 1996, une deuxième phase du projet s'est déroulée avec la pose de huit autres capteurs identiques cette fois-ci sur les parois du même caisson: sept de part et d'autre de la paroi perforée $(z=+1.40,+3.60$ et $+5.60 \mathrm{~m})$ et un sur la paroi pleine de la chambre côté mer $(z=+5.60 \mathrm{~m})$. Ces opérations ont nécessité la participation de l'ensemble du personnel du laboratoire et l'emploi de techniques dérivées de la spéléo.

Enfin un système d'acquisition définitif a été implanté dans le bâtiment de la balise du musoir, en Janvier 1997.

\subsection{Paramètres d'acquisition}

Des sept capteurs implantés en 1991, quatre ne répondent plus et donc seuls les capteurs référencés $\mathrm{r} 1(\mathrm{x}=+1.04 \mathrm{~m}), \mathrm{r} 2(\mathrm{x}=+3.04 \mathrm{~m})$ et $\mathrm{r} 3(\mathrm{x}=+7.04 \mathrm{~m})$ donnent un signal.

Les acquisitions des valeurs de pression sur et sous le caisson se déroulent à intervalles réguliers, de façon autonome. Les résultats présentés proviennent d'enregistrements d'une durée de dix à vingt minutes, à une fréquence d'échantillonnage de trois hertz, entre Janvier et Octobre 1997. La durée des 
acquisitions correspond au passage de soixante dix à quatre vingt vagues en dix minutes.

\subsection{Analyses des pressions}

Nous avons réalisé une analyse temporelle et statistique basée sur la technique du "Zero Up Crossing" et une analyse spectrale.

L'absence d'un houlographe sur le site complique la détermination des paramètres réels de la houle incidente. Ces caractéristiques sont donc évaluées d'après les pressions collectées par les capteurs les plus proches de la surface, à l'extérieur du caisson.

En toute rigueur le signal délivré par un tel capteur est le produit de la superposition de l'onde incidente et de l'onde réfléchie, attaquant la paroi obliquement, pour une profondeur faible. Malgré ces approximations, nous obtenons tout de même des ordres de grandeur de hauteur de houle concordants avec les estimations visuelles du Sémaphore de Dieppe.

\section{Résultats}

A la lumière des premiers résultats, nous pouvons établir deux parties distinctes, la première concernant le radier, la seconde la paroi perforée.

\subsection{Le radier}

\subsubsection{Analyse temporelle}

La théorie classique de calcul des pressions sous un caisson perforé ("uplift pressures") est basée sur une distribution triangulaire de celles-ci. La souspression est maximale au bord amont de la semelle (au sens de la propagation de la houle) et décroît linéairement pour s'annuler au bord de la semelle côté port.

Dans notre cas, comme le montre la figure 2 , nous n'obtenons pas cette répartition puisque les valeurs maximales des pressions restent quasiment constantes pour une distance au mur perforé croissante. Les autres caractéristiques statistiques $\left(\mathrm{P}_{1 / 3}\right.$, Pression moyenne,....) ne subissent pas non plus de variations supérieures à quelques pour-cent.

Cette répartition "rectangulaire" est à rapprocher des résultats obtenus par nos collègues du Centre de Recherche Hydraulique d'ENEL Milan (De Gerloni et al., 1997) pour le caisson perforé multichambre de Porto Torres (Sardaigne). Ils ont en effet mis en évidence une distribution des valeurs maximales des souspressions ne respectant pas non plus la théorie fortement décroissante (figure 3). De plus, au droit de la semelle côté port les valeurs sont non nulles, voire du même ordre que celles présentes sous le caisson. Ce phénomène n'a pas pu être reproduit fidèlement en canal à houle jusqu'à présent, les simulations physiques tendant à suivre la répartition théorique.

Les pressions calculées selon la formule de Takahashi et Shimosako (1994) sont manifestement surestimées. De ce fait lors de la conception de l'ouvrage, les forces de basculement supposées sont elles aussi supérieures à celles calculables à 
partir des sous-pressions réelles (au minimum deux à trois fois). Cette surestimation des valeurs annulerait donc le risque d'accident par basculement, ruine qui par ailleurs ne s'est pas produite sur une digue. En effet jusqu'à présent seuls des glissements ont provoqué la destruction de tels ouvrages.

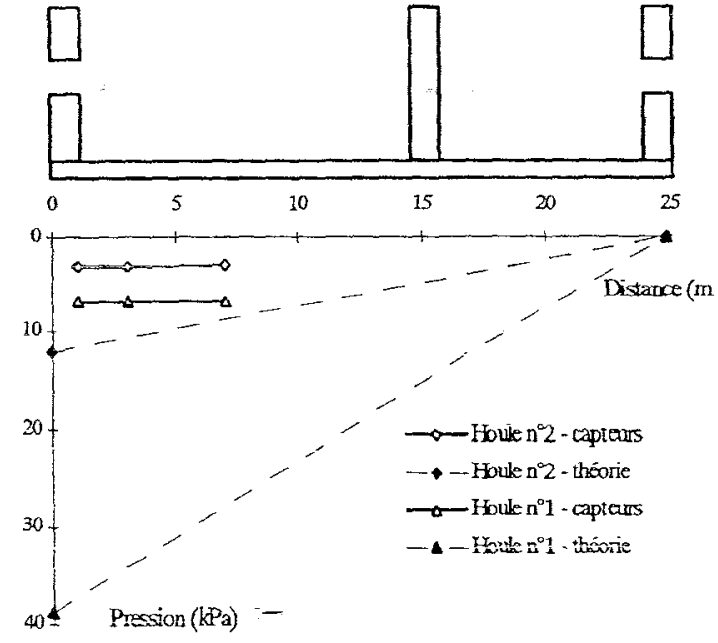

Figure 2 : Répartition des pressions maximales sous le caisson Jarlan de Dieppe

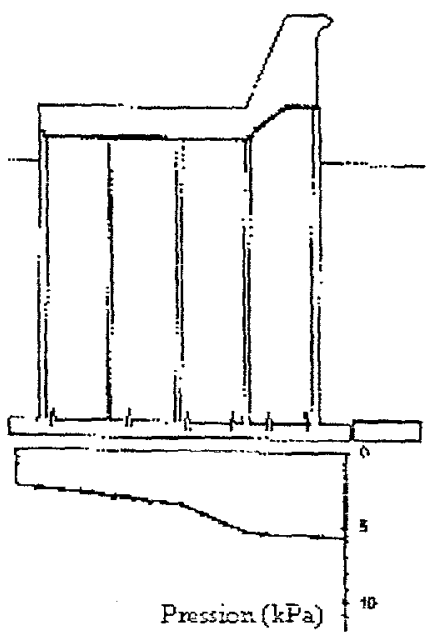

Figure 3 : Répartition des pressions sous le caisson de Porto-Torres

(d'après de Gerloni et al.)

Les variations temporelles des pressions des capteurs du radier sont simultanées et en phase avec celles enregistrées sur la façade perforée.

Le déphasage temporel maximal présent entre les deux capteurs les plus éloignés ( $d=6 \mathrm{~m}$ pour $\mathrm{r} 1$ et $\mathrm{r} 3$ ) est de l'ordre de $0.33 \mathrm{~s}$, valeur qui est liée à la faible fréquence d'acquisition du système. Les vitesses de propagation correspondant à un tel retard seraient alors supérieures à $15 \mathrm{~m} / \mathrm{s}$, valeurs incompatibles avec celles d'écoulements de fluide en milieu poreux. Elles sont aussi supérieures aux vitesses existantes dans la houle à cette profondeur.

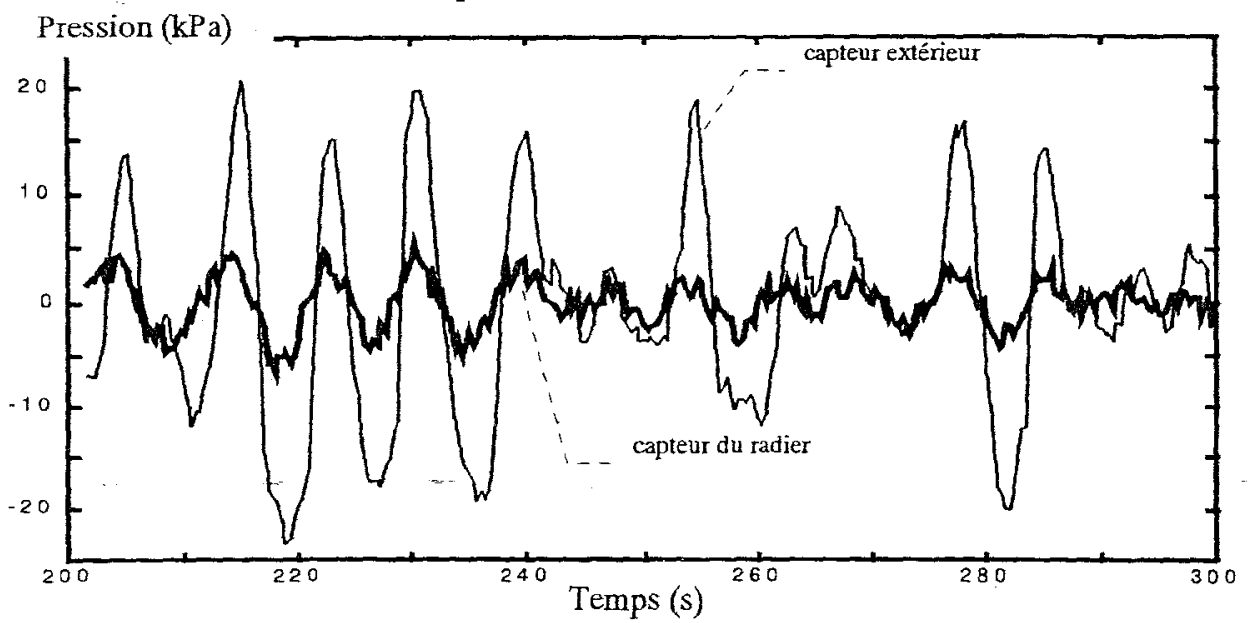

Figure 4 : Variations temporelles des pressions sous le caisson $(x=+1.04 \mathrm{~m})$ et sur la paroi perforée $(\mathrm{z}=+5.60 \mathrm{~m})$. 


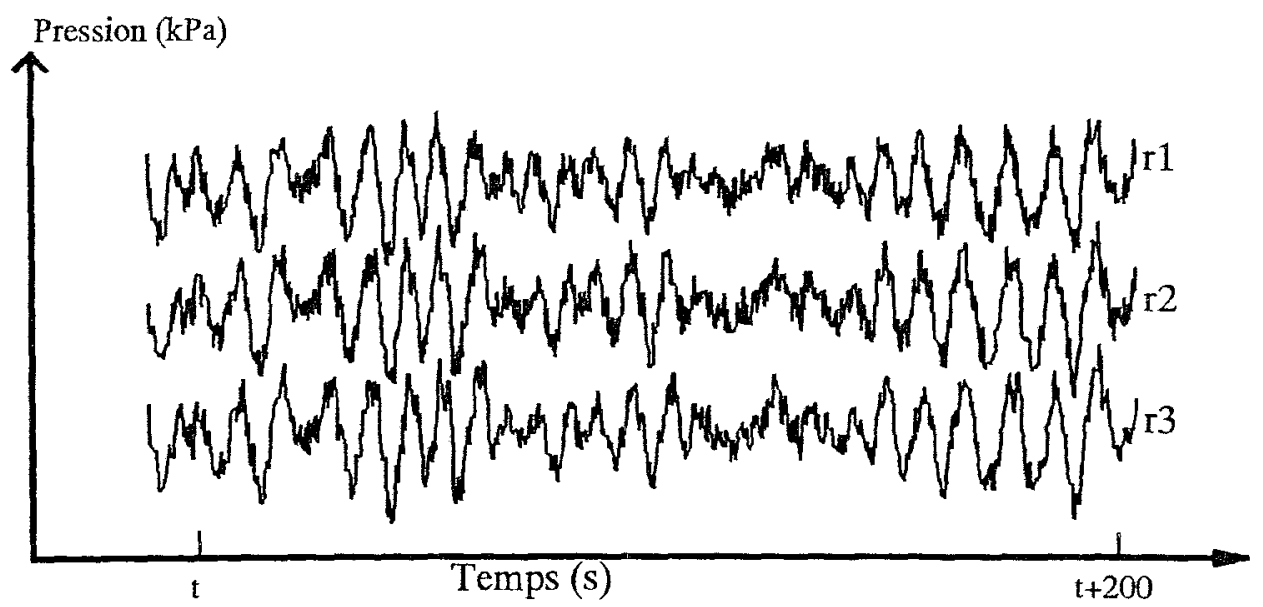

Figure 5: Evolution temporelle comparée des pressions des trois capteurs du radier (les échelles de pression sont identiques mais décalées pour la lisibilité).

\subsubsection{Analyse spectrale}

L'analyse spectrale des signaux des capteurs du radier montre que les fondations filtrent la houle. Comme le montre la figure 6, l'onde incidente est composée de fréquences basses correspondantes à des houles d'Atlantique qui se propagent dans la Manche et de fréquences plus élévées provenant de la mer du vent locale. D'une façon générale les périodes inférieures à 8.5 - 7.5 secondes sont très atténuées voire inexistantes dans le radier alors que les périodes supérieures à ce seuil sont transmises. Les fondations semblent donc se comporter comme un filtre passe-bas.

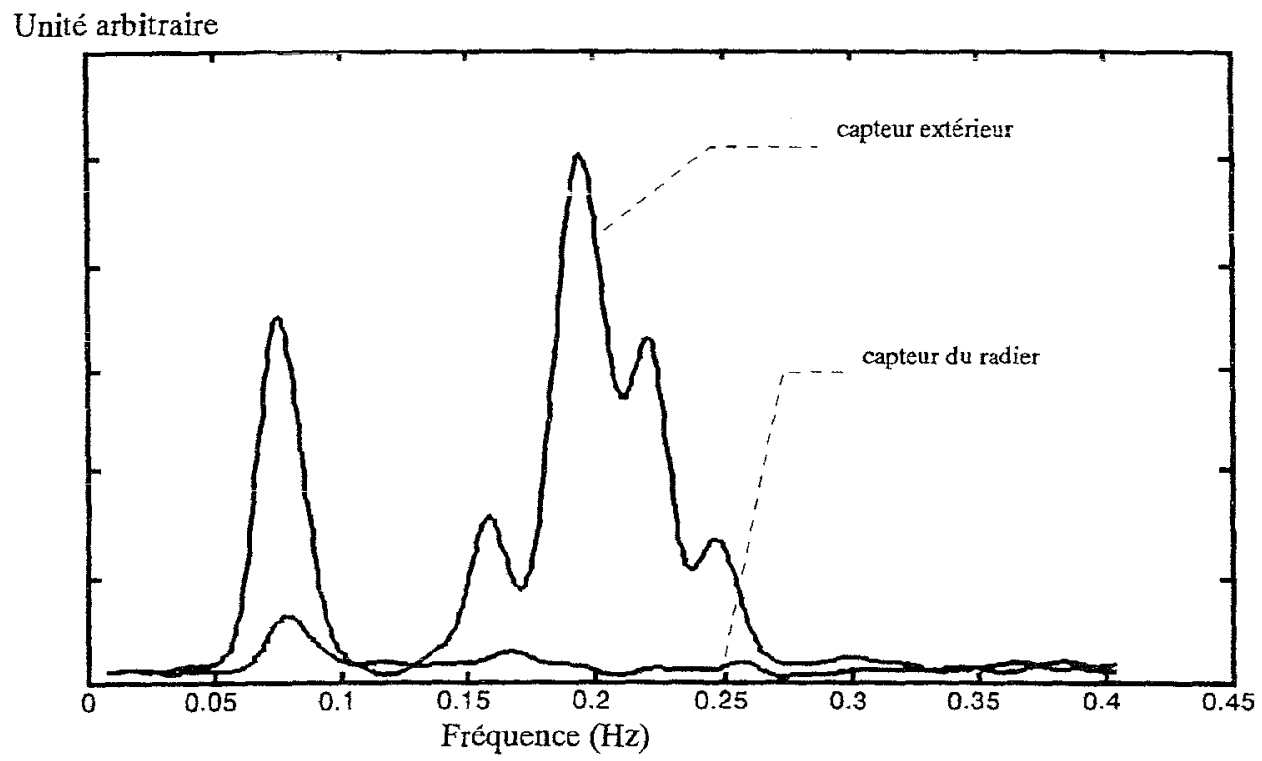

Figure 6 : Comparaison entre les spectres des signaux d'un capteur extérieur de la paroi perforée $(\mathrm{z}=+5.60 \mathrm{~m})$ et d'un capteur du radier $(\mathrm{x}=+1.04 \mathrm{~m})$ 


\subsection{La paroi perforée}

\subsubsection{Analyse temporelle}

La figure 7 présente une séquence d'un enregistrement des pressions lors d'une tempête pour des capteurs de part et d'autre de la paroi perforée, pour une même altitude. Dans les cas les plus défavorables, il existe un déphasage variable entre les deux signaux. Le retard constaté prouve que peut exister un pic de pression sur la façade extérieure pour une pression nulle de l'autre côté.

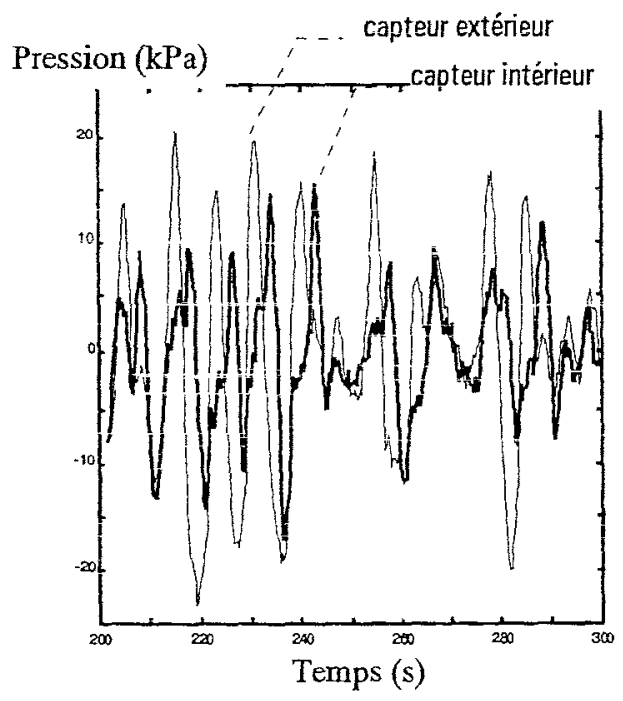

Figure 7 : Evolution temporelle des pressions de part et d'autre de la paroi perforée $(z=+5.60 \mathrm{~m})$

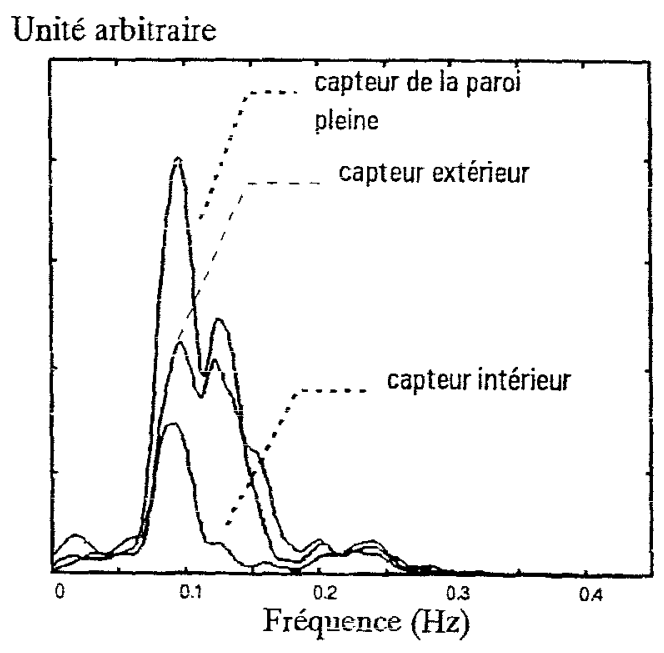

Figure 8 : Comparaison des spectres des signaux sur les différentes parois du caisson $(\mathrm{z}=+5.60 \mathrm{~m})$

Durant le même enregistrement, sur cette la face externe, des pressions inférieures au niveau moyen ont lieu alors que la pression exercée sur la face interne est maximale. Nous nous trouvons alors dans des conditions exceptionnelles où la pression résultante maximale appliquée à la paroi sera dirigée vers l'extérieur du caisson et non pas dans la direction de propagation de la houle.

Ce phénomène important correspond à une différence de hauteur d'eau entre l'extérieur et l'intérieur. Il semblerait donc être un mécanisme à prendre en compte lors des calculs du dimensionnement.

Si l'agitation est essentiellement due aux composantes basses fréquences des houles atlantiques, il n'existe pas de déphasage notable entre les deux faces de la paroi perforée. Le fait que les Iongueurs d'ondes soient très importantes par rapport aux dimensions de la chambre explique que leur propagation ne soit pas véritablement perturbée par le franchissement du mur. 


\subsubsection{Analyse spectrale}

La comparaison des spectres des pressions enregistrées de part et d'autre de la paroi perforée indique que celle-ci se comporte comme un filtre. Certaines fréquences (périodes de l'ordre de six à huit secondes) sont totalement absentes des signaux derrière le mur. Toutefois elles sont présentes sur le mur plein laissant supposer une recombinaison de la houle incidente au sein de la chambre.

Au contraire, des périodes d'environ quatre secondes sont toujours présentes dans les signaux des capteurs proches de la surface, en particulier à l'intérieur de la chambre comme le montre la figure 8. D'après les dimensions du caisson, notamment sa largeur de chambre, ces types de périodes correspondraient à des périodes de résonance.

Nous ne pouvons pas pour l'instant caractériser le rôle du filtre représenté par la paroi perforée (passe-bas ou stop-bande) sans connaître les paramètres de la houle au large. En effet il nous faut déterminer si les périodes particulières de résonance existent dans l'onde incidente ou si elles apparaissent dans la chambre et diffusent ensuite par les trous. De même il est possible que des harmoniques se développent et perturbent cette analyse.

Il est par contre certain que ce filtrage des fréquences constitue une caractéristique importante du fonctionnement du caisson.

\section{Conclusions}

Le dimensionnement des caissons perforés est déterminé par des formules semiempiriques essentiellement calibrées par la modélisation. Il est donc nécessaire de valider ces calculs par la comparaison avec des mesures réalisées in situ. Les valeurs des pressions exercées sur un caisson Jarlan de la digue de Dieppe représentent donc la possibilité de connaître les forces existantes sur une telle structure.

Ces pressions ont des modules inférieurs à ceux prédits par la théorie mais ont aussi des répartitions moins caractéristiques que prévues aussi bien pour les fondations que pour les parois. De plus nous avons établi que les comportements de l'ensemble talus-caisson d'une part, et de la paroi perforée d'autre part, soñt dépendants des fréquences des houles incidentes.

Il nous est nécessaire de déterminer l'origine de l'ébranlement enregistré sous le caisson: mouvement vertical de pilonnement de l'ensemble du caisson sous l'effet de l'agitation de la masse d'eau dans les chambres ou talus se comportant comme un guide piégeant une onde entre la roche et la semelle du caisson.

Un deuxième axe d'étude est constitué par la recherche des relations entre les valeurs des pressions enregistrées et les paramètres de la houle incidente. Ceci sera rendu possible par l'exploitation des données fournies par un houlographe Inter Ocean S4DW dont la pose est prévue en début d'année 1998.

Ces résultats confirment l'intérêt de l'instrumentation d'éléments de digues. Ils nous permettent ainsi d'espérer fournir des indications pertinentes aux concepteurs 
des digues pour les futures règles de calcul mais aussi pour la mise au point de nouveaux codes numériques plus complexes.

Les études réalisées sur le caisson Jarlan sont soutenues par le programme de recherche européen MAST III Proverbs, avec la collaboration de la DDE de Dieppe et le STCPMVN. Nous remercions également Messieurs Arsié, Lambert et Vasselin pour leur aide lors de la pose des capteurs des parois.

\section{Références}

Goda, Y. (1985): Random seas and design of maritime structures. Tokyo: University of Tokyo Press,

de Gerloni, M. et al. (1997): Scale effects in uplift pressure measurements on caisson breakwaters. Proceedings of Las Palmas Worshop. MAST III Proverbs.

Takahashi, S. et Shimosako, K. (1994): Wave pressure on a perforated wall caisson. Hydro'Port 94

Miègeville, C. (1996): Etude du caisson Jarlan. Projet de fin d'études. ESTP Cachan. 